\title{
Metastatic Spermatic Cord Tumor From Colorectal Cancer
}

\author{
Ji Geon Jang, Hye Yun Jeong, Ki Soo Kim, Mi Jung Park, Jin Sook Lee ${ }^{1}$, Sang Su Kim², Ho Young Kim³ \\ Departments of Internal Medicine, ${ }^{1}$ Pathology, ${ }^{2}$ Radiology, and ${ }^{3}$ Surgery, Good Samsun Hospital, Busan, Korea
}

Metastatic tumors of the spermatic cord are extremely rare, and the prognosis for patients is typically poor. In the majority of cases, the primary tumor occurs in the gastrointestinal tract. We report a case of a 62-year-old man with a metastatic spermatic cord tumor. The patient complained of groin discomfort with a tender mass in the right inguinal area. An excisional biopsy was performed, and the pathologic finding was a metastatic mucinous adenocarcinoma. We performed a systemic evaluation including colonoscopy, abdominal computed tomography, and total-body positron emission tomography, and the primary tumor was confirmed to involve the total colon, including the cecum, sigmoid colon, and rectum. The pathologic finding for rectum revealed a mucinous adenocarcinoma compatible with a metastatic spermatic cord tumor.

\section{Keywords: Spermatic cord; Neoplasm metastasis; Colorectal neoplasms}

\section{INTRODUCTION}

A spermatic cord tumor is unusual, and, in particular, a metastatic tumor of the spermatic cord is very rare $[1,2]$. The primary lesion of a metastatic spermatic cord tumor is known to involve the gastrointestinal system, the kidneys, and the prostate $[1,3,4]$. The liver and the lungs are usual sites for the metastasis of colorectal cancer, and colorectal cancer has been reported to spread to various organs such as the peritoneum and the lymph nodes [5]. However, colorectal cancer very rarely generates a metastatic lesion in the spermatic cord $[1,3,4,6-10]$. The authors report their experience with a patient in whom colon cancer was confirmed by the discovery of a metastatic spermatic cord tumor.

\section{CASE REPORT}

A male patient, 62 years old, visited Good Samsun Hospital with a chief complaint of a right inguinal region mass accompanied by pain for the previous 3 months. He had no special history of dis-

Received: June 10,2015 - Accepted: August 18, 2015

Correspondence to: Mi Jung Park, M.D.

Department of Internal Medicine, Good Samsun Hospital, 326 Gaya-daero, Sasang-gu, Busan 47007, Korea

Tel: +82-51-310-9122, Fax: +82-51-313-2367

E-mail: mjpark520@hanmail.net

(C) 2015 The Korean Society of Coloproctology

This is an open-access article distributed under the terms of the Creative Commons Attribution NonCommercial License (http://creativecommons.org/licenses/by-nc/3.0) which permits unrestricted noncommercial use, distribution, and reproduction in any medium, provided the original work is properly cited. ease; however, the year before his visit to our hospital, he had undergone hernia surgery due to an indolent fixed mass in the right inguinal region. On physical examination, his vital signs were stable, and a hard, fixed mass, accompanied by oppressive pain, with a size of $1 \mathrm{~cm} \times 1 \mathrm{~cm}$ was felt in the right inguinal region. The patient's progress was observed for a month during which time an anti-inflammatory analgesic drug was administered to distinguish the oppressive pain from that which might have been generated by the previous hernia surgery. The biopsy of the spermatic cord showed a metastatic mucinous adenocarcinoma (Fig. 1A). The image showed a thickening of the right spermatic cord, and infiltration around soft tissues was confirmed by using the scrotal sonography (Fig. 2). In addition, abdominal computed tomography indicated infiltration of the proximal sigmoid colon and rectum, as well as a diffuse peritoneal seeding metastasis (Fig. 3A, B). A tumor accompanied by redness was observed in the rectum during the colonoscopy, and a core needle biopsy was conducted on that tumor. A mucinous adenocarcinoma that was identical to tissue previously taken from the spermatic cord was diagnosed based on the biopsy result (Fig. 1B). Positron emission tomography (PET) (Fig. 4) confirmed infiltration of the sigmoid colon, rectum, and cecum.

This patient received four rounds of FOLFOX (oxaliplatin with fluorouracil and folinic acid) anticancer chemotherapy, and the right spermatic cord mass was smaller on the abdominal computed tomography that that was done 10 weeks after the diagnosis of a spermatic cord tumor. However, the tumor sizes in the sigmoid colon and the rectum had not changed, and the possibility of a new seroperitoneum was checked. An exploratory laparot- 

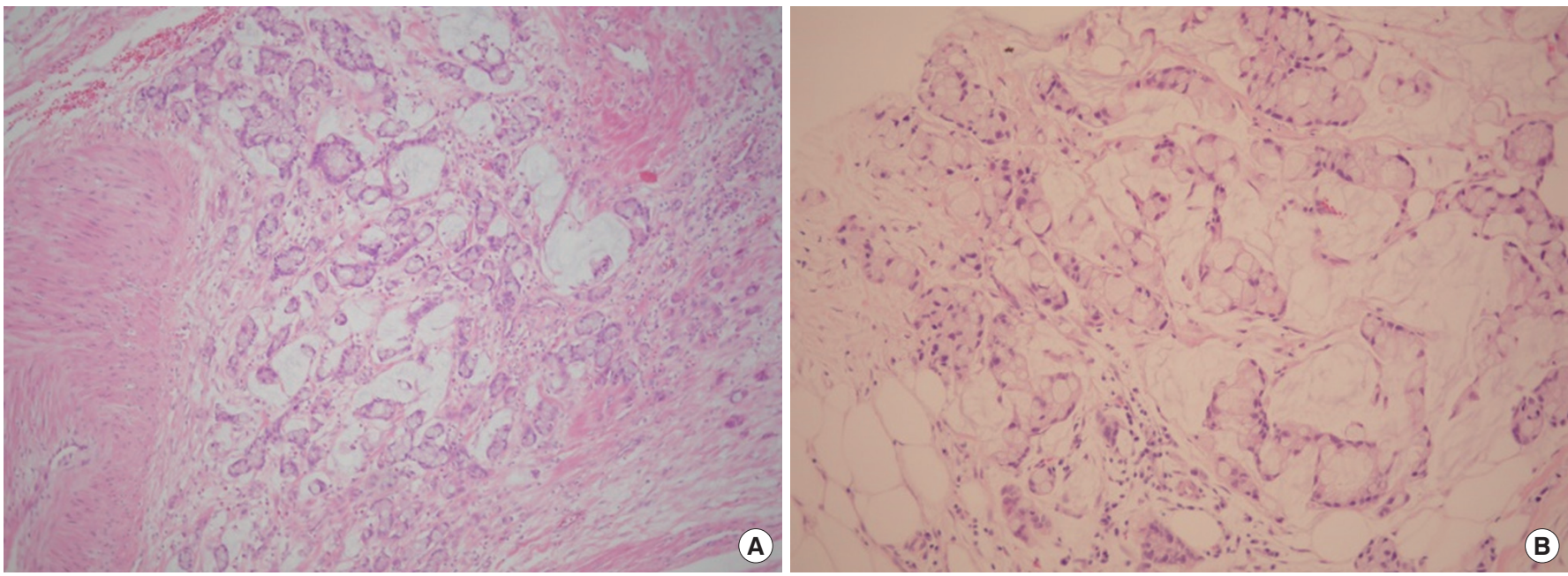

Fig. 1. (A) Microscopic finding for the right spermatic cord shows a mucinous adenocarcinoma (H\&E, $\times 100)$. (B) Microscopic finding for the rectum shows a mucinous adenocarcinoma $(\mathrm{H} \& \mathrm{E}, \times 200)$.

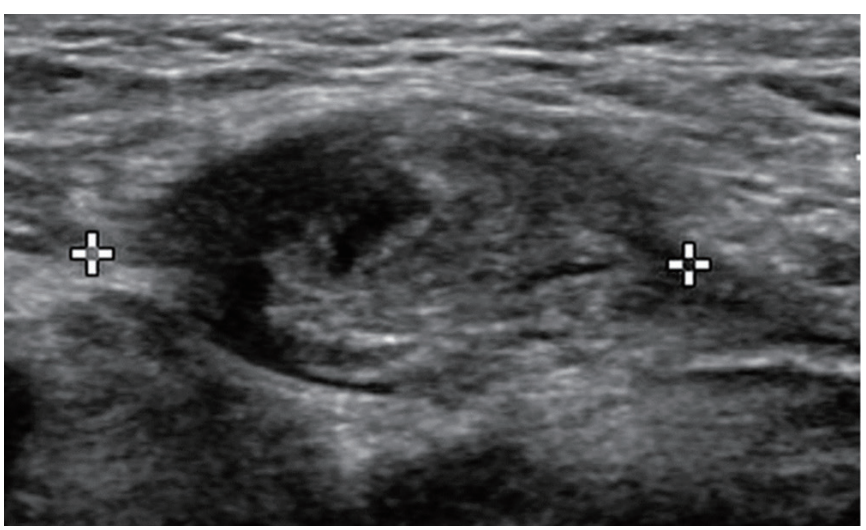

Fig. 2. Ultrasound findings for the right spermatic cord show diffuse thickening with soft tissue infiltration.
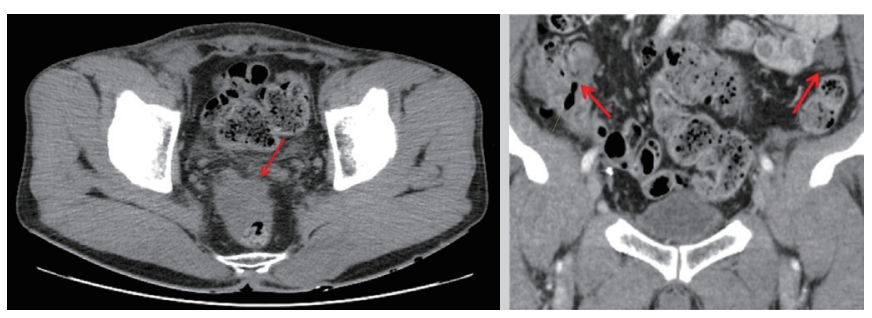

Fig. 3. Computed tomography shows a speculated, rim-enhanced mass in the sigmoid colon and peritoneal seeding nodules (arrow).

omy was conducted to confirm the possibility of salvage surgery to remove the primary lesion from the large intestine, but only an orchiectomy on the right side was completed because its metastasis in the peritoneum, omentum, and small bowel mesentery was extensive. Upon surgery, a broad metastasis in the peritoneum, omentum, and small bowel mesentery was confirmed (Peritoneal
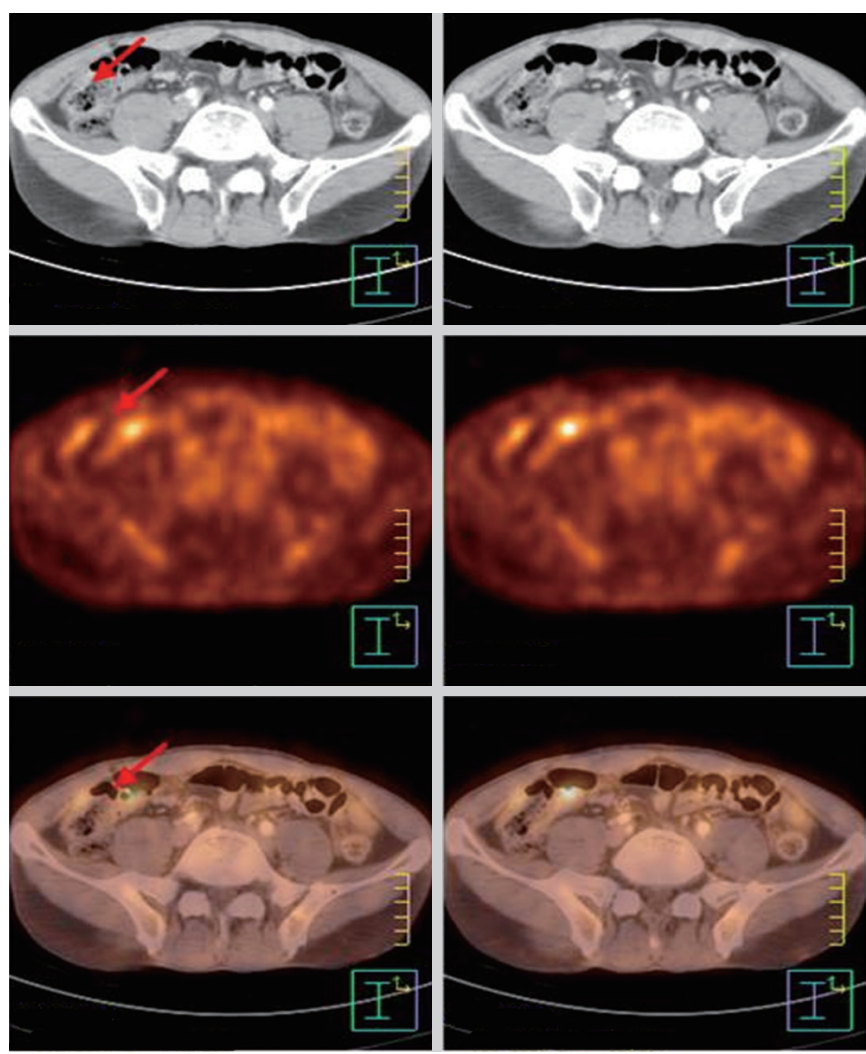

Fig. 4. Positron emission tomography shows an unusual fluorodeoxyglucose uptake in the cecum serosa (arrows).

Cancer Index score, 22), and the biopsy of the peritoneum, omentum, and small bowel mesentery showed a metastatic mucinous adenocarcinoma. As a result of this diagnosis, the therapy was changed to include palliative FOLFIRI (irinotecan with fluoroura- 
cil and folinic acid) chemotherapy. The patient came down with pneumonia after the 3rd FOLFIRI treatment. Antibiotic treatment was started, but the pneumonia proceeded to sepsis within a week. The patient finally passed away three months after the initial diagnosis.

\section{DISCUSSION}

A metastatic tumor of the spermatic cord is very rare $[1,2]$. The primary lesion of this tumor is in the gastrointestinal tract, and in some cases, it is in the kidneys and prostate $[1,3,4]$. The most involved parts in the gastrointestinal tract are the stomach, colon, anus, and pancreas in order of decreasing incidence rate. The frequencies of occurrence in the colon on the right side, the left side, and both sides are reported to be $57.4 \%, 27.8 \%$, and $12.9 \%$, respectively [1]. In this case, PET confirmed infiltration into the sigmoid colon and the rectum.

About $23.8 \%$ of the patients with a metastatic spermatic cord tumor report no symptoms, and most cases are confirmed as the primary lesion progresses [3]. Thus, in most previous cases, the presence of such a tumor was confirmed during therapy for the primary tumor. However, in this case, colon cancer was found due to the discovery of the metastatic spermatic cord tumor.

Until now, five cases of a metastatic spermatic cord tumor have been reported in Korea: three cases for stomach cancer, one case for colon cancer, and one case for esophageal cancer in the gastrointestinal tract $[6,11-13]$. In many cases, metastasis of the spermatic cord presents as multiple metastases into various organs, so its prognosis is very poor, with an average survival of only 9.1 months [3]. The patient in this case report passed away in only 3 months, a survival time much shorter than the reported average survival time.

Colorectal cancer primarily spreads to the lymph nodes, liver, lungs, and brain [5] and very rarely spreads to the spermatic cord or the scrotum $[1,3,4,6-10]$. The mechanism for metastasis to the spermatic cord in patients with colon cancer is not clear. However, various routes have suggested $[4,7,8]$ : infiltration through the lymph nodes, passage through the blood vessel, and a reversely go through the spermiduct or possible direct infiltration by the tumors through the walls of the spermiduct [4]. If spread in the peritoneum, a transperitoneal seeding is also available through the patent processus vaginalis $[7,8]$. Even in this case, no direct connection of the masses in the abdominal cavity was observed. All metastasis possibilities, as confirmed through the peritoneum and the lymph nodes, should be considered for patients with a previous history of hernia surgery. No correlation with the metastatic spermatic cord tumor was revealed in this case, but the patient had a history of surgery due to an inguinal hernia. Physiological tissue tests on the hernia pocket after surgery have been reported to show a $0.4 \%$ incidence of a malignant tumor [10], and a case in which a metastatic spermatic cord tumor was mistaken for a hernia has also been reported [12].
The metastatic spermatic cord tumors reported in both domestic and foreign literature were almost always discovered while treating the original cancer or observing its progress. As in this case, however, the primary lesion may be discovered by finding the spermatic cord lesion before finding the original cancer. Therefore, a mass in the inguinal region should always be considered as a malignant tumor. In addition, if an inguinal hernia is found in elderly patients, the possibility of a metastatic tumor should be considered, which should be confirmed by conducting a histopathological examination.

\section{CONFLICT OF INTEREST}

No potential conflict of interest relevant to this article was reported.

\section{REFERENCES}

1. Kanno K, Ohwada S, Nakamura S, Ohya T, Iino Y, Morishita Y, et al. Epididymis metastasis from colon carcinoma: a case report and a review of the Japanese literature. Jpn J Clin Oncol 1994;24: 340-4.

2. Beccia DJ, Krane RJ, Olsson CA. Clinical management of nontesticular intrascrotal tumors. J Urol 1976;116:476-9.

3. Algaba F, Santaularia JM, Villavicencio H. Metastatic tumor of the epididymis and spermatic cord. Eur Urol 1983;9:56-9.

4. Monn L, Poticha SM. Metastatic tumors of spermatic cord. Urology 1975;5:821-3.

5. Kuba H, Sato N, Uchiyama A, Nakafusa Y, Mibu R, Yoshida K, et al. Mediastinal lymph node metastasis of colon cancer: report of a case. Surg Today 1999;29:375-7.

6. Kim HJ, Moon EJ, Kang WK, Hong SH, Jung CK, Oh ST. Sigmoid colon cancer with metastasis to the right spermatic cord. J Korean Soc Coloproctol 2007;23:203-5.

7. Ney C, Roth JJ, Lopez CA. Carcinoma of the prostate with metastases to the testicle. Arch Surg 1959;79:1028-31.

8. Cricco RP, Kandzari SJ. Secondary testicular tumors. J Urol 1977;118:489-90.

9. Obrand DI, Gordon PH. Incidence and patterns of recurrence following curative resection for colorectal carcinoma. Dis Colon Rectum 1997;40:15-24.

10. Yoell JH. Surprises in hernial sacs; diagnosis of tumors by microscopic examination. Calif Med 1959;91:146-8.

11. Kim MK, Kim HS, Cheon SY, Kim HJ, Jeong YB, Kim YG. Metastatic tumor of the spermatic cord from gastric cancer. Korean J Urol 2003;44:105-7.

12. Han KS, Kim SS, Kim HJ, Shin EA, Choi JS, Rha KH. Spermatic cord metastasis of esophageal cancer, mimicking as an incarcerated hernia. Korean J Urol 2005;46:197-9.

13. Kim JH, Kim DS, Cho HD, Lee MS. Late-onset metastatic adenocarcinoma of the spermatic cord from primary gastric cancer. World J Surg Oncol 2014;12:128. 